\title{
Exact solution for the conjugate fluid-fluid problem in the thermal entrance region of laminar counterflow heat exchangers
}

\author{
Marcos Vera, Amable Liñán
}

A B S T R A C T

\begin{abstract}
A generalized Lévêque solution is presented for the conjugate fluid-fluid problem that arises in the ther-mal entrance region of laminar counterflow heat exchangers. The analysis, carried out for constant prop-erty fluids, assumes that the Prandtl and Peclet numbers are both large compared to unity, and neglects axial conduction both in the fluids and in the plate, assumed to be thermally thin. Under these conditions, the thermal entrance region admits an asymptotic selfsimilar description where the temperature varies as a power $j$ of the axial distance, with the particularity that the selfsimilarity exponent must be deter-mined as an eigenvalue by solving a transcendental equation arising from the requirement of continuity of heat fluxes at the heat conducting wall. Specifically, the analysis reveals that $j$ depends only on the lumped parameter ${ }^{\wedge}=\left(\mathcal{A}_{2} / \mathcal{A}_{1}\right)^{1 / 3}\left(\alpha_{1} / \alpha_{2}\right)^{1 / 3}\left(k_{2} / k_{1}\right)$, defined in terms of the ratios of the wall velocity gra-dients, $\mathcal{A}_{i}$, thermal diffusivities, $\alpha_{i}$, and thermal conductivities, $k_{i}$, of the fluids entering, 1 , and exiting, 2 , the heat exchanger. Moreover, it is shown that for large (small) values of ${ }^{\wedge}$ the solution reduces to the classical first (second) Levêque solution. Closed-form analytical expressions for the asymptotic temper-ature distributions and local heat-transfer rate in the thermal entrance region are given and compared with numerical results in the counterflow parallel-plate configuration, showing very good agreement in all cases.
\end{abstract}

\section{Introduction}

The canonical problem of the steady-state convective heat transfer in the thermal entrance region of a circular tube - or flat channel - with a fully developed laminar velocity profile is commonly known as the Graetz-Nusselt problem [1-3]. This fundamental problem, and the analogous mass-transfer process, have received a great deal of attention over the past decades due to their importance in heating and cooling equipment, as well as in chemical, pharmaceutical, food-processing, and many other industrial applications. A comprehensive review of the relevant literature may be found in classical textbooks on convective heat transfer [4-7]. However, we shall give here a brief account of the most relevant references related to the present work.

The analytical solution of the Graetz-Nusselt problem involves separation of variables followed by eigenfunction expansions. The resulting expansion is convergent for all axial positions downstream of the inlet, but the convergence becomes very slow at small axial distances, where a large number of terms is required for an accurate description of the solution. Thus, as the inlet is approached the cal- culation becomes tedious and the numerical solution may not even converge, so that an alternative procedure is required.

The alternative approach, due to Lévêque [8], exploits the fact that for sufficiently large Peclet numbers the temperature variations are initially confined to a thin layer of fluid adjacent to the wall, where the velocity profile is linear - corresponding to the near-wall velocity gradient of the fully developed flow. The first extension to the problem involved the replacement of the isothermal wall assumption by a constant heat flux, leading to the socalled second Lévêque problem [9]. The solution of the thermal entrance problem was then expanded as an asymptotic series for small axial distances - the leading order term being Lévêque's solution - to include nonlinear inlet velocity profiles and curvature effects [10-13]. The analysis was further extended to account for non-axisymmetric heat transfer [14], unsteady effects [15], nonNewtonian fluids of different rheologies with viscous dissipation [16-19], wall slip [20], and swirling flows [21]. Other authors resorted to similar techniques to study the thermal entrance region of concentric annuli [22-24]. External convection effects were also included as a third kind of boundary condition [25], in which the heat flux normal to the wall is assumed to be proportional to the wall temperature.

Regarding conjugate fluid-solid problems [26], the analysis of the thermal entrance region was generalized to investigate the 


\section{Nomenclature}

A expansion coefficient.

$a_{i} \quad$ channel half-width of fluid $i$.

$C_{n} \quad$ expansion coefficient corresponding to the $n$th eigenfunction.

specific heat of fluid $i$.

$n$th eigenfunction.

dimensionless parameter, $\left(a_{1} k_{2}\right) /\left(a_{2} k_{1}\right)$.

thermal conductivity of fluid $i$.

lumped parameter, $m^{1 / 3} k$.

length of the heat-exchanger.

dimensionless parameter, $\left(a_{2} \mathrm{Pe}_{2}\right) /\left(a_{1} \mathrm{Pe}_{1}\right)$.

mass flow rate of fluid $i, 2 a_{i} V_{i} \rho_{i}$.

Peclet number of fluid $i, 2 a_{i} V_{i} / \alpha_{i}$.

Prandtl number of fluid $i, v_{i} / \alpha_{i}$.

temperature.

longitudinal velocity of fluid $i$.

average flow velocity of fluid $i$.

Whittaker's function, $W_{\kappa, \mu}(z)$.

longitudinal distance from the inlet of fluid 1.

transverse distance from channel $i$ symmetry plane.

dimensionless transverse coordinate, $Y_{i} / a_{i}$.

\section{Greek letters}

$\alpha_{i} \quad$ thermal diffusivity of fluid $i, k_{i} /\left(\rho_{i} c_{i}\right)$.

$\Gamma \quad$ Gamma function, $\Gamma(z)$.

$\kappa \quad$ first argument of Whittaker functions.

$\lambda_{n} \quad n$th eigenvalue.

$\mu \quad$ second argument of Whittaker functions.

$v \quad$ dimensionless local heat-transfer rate, $\partial \theta_{1} /\left.\partial y_{1}\right|_{y_{1}=1}$.

$v_{i} \quad$ kinematic viscosity of fluid $i$

$\rho_{i} \quad$ density of fluid $i$

$\theta_{i} \quad$ normalized temperature of fluid $i$.

$\xi$ dimensionless longitudinal coordinate, $X /\left(\mathrm{Pe}_{1} a_{1}\right)$

Subscripts

$i \quad$ subscript used indistinctly for fluids 1 and 2 .

in Inlet.

$L \quad$ length of the heat-exchanger.

$m$ bulk, or mixing-cup, temperature.

out outlet.

$w \quad$ heat-exchanging wall effect of the wall thermal resistance considering both isothermal and constant heat flux boundary conditions at the outer wall surface [27-35]. Fluid-fluid problems have also received great attention due to their relevance for the design of practical heat transfer systems, such as double-pipe, or multilayered, heat exchangers. Specifically, several investigations have been reported dealing with both the co-current [36-40] and counter-current [41-49] flow configurations. In these studies the counter-current configuration typically results in a more challenging mathematical problem, since due to the existing counterflow pattern the set of governing equations is elliptical rather than parabolic even though longitudinal conduction effects are not taken into account.

Besides the classical approach, based on a combination of theoretical, asymptotic, and numerical methods, modern mathematical tools have recently been incorporated into the study of the GraetzNusselt problem. Thus, the analysis of the steady-state heat transfer in the thermal entrance region of circular tubes [50], in microchannels formed by parallel-plates [51], or in multilayered parallel-plate heat exchangers [52], has benefited from the valuable help of symbolic algebraic computation. Moreover, an interesting application of the method of lines resulting in the amplification of the size of Lévêque's thermal entrance subregion has also been reported [53,54]. Finally, an alternative approach based on Laplace's transform can be found in $[55,56]$.

Although several studies have been presented in the literature, no analytical results have been found for the asymptotic behavior of the temperature field in the thermal entrance region of conjugate counter-current fluid-fluid problems. Thus, the aim of this paper is to combine analytical, symbolic, and numerical tools to extend the classical Lévêque analysis to the conjugate fluid-fluid problem that arises in the thermal entrance region of laminar counterflow heat exchangers. Specifically, the analysis will shed new light of the singular nature of the temperature field for small axial distances in the relevant limit of large Prandtl and Peclet numbers with negligible wall thermal resistance.

The paper is organized as follows. In Section 2 we present the mathematical formulation of the problem. In Section 3 we particularize the formulation to the thermal entrance region at small axial distances, showing that in this region the temperature field admits an asymptotic self-similar solution of the second kind. In Section 4 we write closed form analytical expressions for the self-similar solution in terms of Whittaker's functions. The resulting expressions for the temperature distributions and local heat-transfer rate are discussed and compared with fully numerical simulations in Section 5. Finally, the conclusions are presented in Section 6.

\section{Problem formulation}

As a convenient framework for the analysis of the thermal entrance region in laminar counterflow heat exchangers, we shall analyze the laminar flow of two constant-property Newtonian fluids through a multilayered counterflow parallel-plate heat exchanger composed by a relatively large number of channels separated by thermally thin plates. The conducting plates allow the exchange of heat through a section of length $L$, presenting insulated sections at both ends of the heat exchange section, where no heat transfer is allowed. In the counterflow configuration considered here, the two fluids, denoted by 1 and 2 (the subscript $i=1,2$ will be used indistinctly for both fluids), flow in opposite directions in adjacent channels.

Fig. 1 shows a sketch of the physical model under study, where $X$ denotes the longitudinal (i.e. axial) coordinate measured from the inlet of fluid 1 , and $Y_{i}$ denotes the transverse coordinate measured from the symmetry plane of channel $i$. The Prandtl number, $\operatorname{Pr}_{i}=v_{i} / \alpha_{i}$, defined in terms of the kinematic viscosity, $v_{i}$, and thermal diffusivity, $\alpha_{i}$, of fluid $i$, will be assumed to be large compared to unity, so that the thermal entry length is large compared to the hydrodynamic entry length, a good approximation for most nonmetallic liquids in applications. Then, as long as the flow remains laminar, it can be assumed to be a fully developed Poiseuille flow, $u_{i}\left(Y_{i}\right)= \pm(3 / 2) V_{i}\left[1-\left(Y_{i} / a_{i}\right)^{2}\right]$, where $a_{i}$ denotes the channel halfwidth corresponding to fluid $i$, and the plus (minus) sign holds for fluid 1 (2).

In addition, we shall assume that the Peclet numbers of the flow in the channels, $\mathrm{Pe}_{i}=2 a_{i} V_{i} / \alpha_{i}$, are both large compared to unity. In this case axial heat conduction in the fluids is confined to small regions located at the end sections of the heat exchanger, close to the heat conducting plates, whose characteristic size, of order $\left[\alpha_{i} a_{i} /\left(2 V_{i}\right)\right]^{1 / 2}=\mathrm{Pe}_{i}^{-1 / 2} a_{i}$, is small compared to $a_{i}$ when $\mathrm{Pe}_{i} \gg 1$. In 


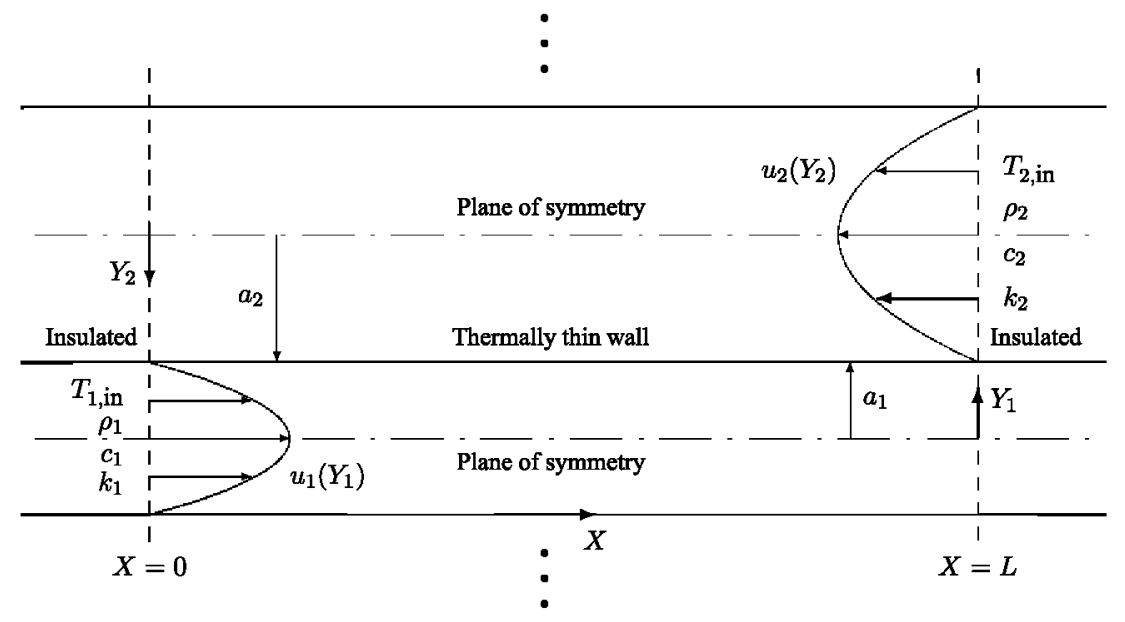

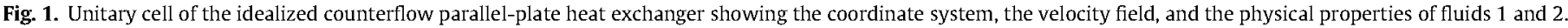
The domain under study is shaded gray.

this limiting case we can neglect axial conduction in the fluid as a first approximation, and therefore we shall assume that the inlet temperature of fluid $i$ is uniform and equal to $T_{i, \text { in }}[43,57]$.

To write the problem in non-dimensional form, we introduce the dimensionless variables $\xi=X /\left(\mathrm{Pe}_{1} a_{1}\right), y_{i}=Y_{i} / a_{i}$, and $\theta_{i}=(-$ $\left.T_{i}-T_{1, \text { in }}\right) /\left(T_{2, \text { in }}-T_{1, \text { in }}\right)$. Then, under the assumptions stated above, the energy equations for fluids 1 and 2 reduce, as a first approximation, to a balance between axial convection and transverse conduction

$\frac{3}{4}\left(1-y_{1}^{2}\right) \frac{\partial \theta_{1}}{\partial \xi}=\frac{\partial^{2} \theta_{1}}{\partial y_{1}^{2}}$,

$-m \frac{3}{4}\left(1-y_{2}^{2}\right) \frac{\partial \theta_{2}}{\partial \xi}=\frac{\partial^{2} \theta_{2}}{\partial y_{2}^{2}}$

in $0<\xi<\xi_{L}, 0<y_{i}<1$, where the parameters $m=a_{2} \mathrm{Pe}_{2} /\left(a_{1} \mathrm{Pe}_{1}\right)$ and $\xi_{L}=L /\left(a_{1} \mathrm{Pe}_{1}\right)$ are both assumed to be of order unity.

The above equations have to be integrated with the boundary conditions at the channel symmetry planes

$\frac{\partial \theta_{1}}{\partial y_{1}}=\frac{\partial \theta_{2}}{\partial y_{2}}=0 \quad$ at $0<\xi<\xi_{L}, \quad y_{i}=0$,

at the heat conducting wall

$$
\begin{aligned}
& \theta_{1}=\theta_{2} \equiv \theta_{w}(\xi) \\
& \frac{\partial \theta_{1}}{\partial y_{1}}=-k \frac{\partial \theta_{2}}{\partial y_{2}} \equiv v(\xi)
\end{aligned} \quad \text { at } \quad 0<\xi<\xi_{L}, \quad y_{i}=1,
$$

and at the inlet sections

$\theta_{1}=\theta_{1, \text { in }}=0$ at $\xi=0, \quad 0 \leqslant y_{1}<1$,

$\theta_{2}=\theta_{2, \text { in }}=1$ at $\xi=\xi_{L}, \quad 0 \leqslant y_{2}<1$

Notice that the requirement of continuity of heat fluxes at the heat conducting wall, given by Eq. (4), introduces the additional parameter $k=\left(a_{1} k_{2}\right) /\left(a_{2} k_{1}\right)$. Note also that in the thermally thin wall regime considered here the absence of longitudinal heat conduction effects for $\mathrm{Pe}_{i} \gg 1$ implies that the temperature at both ends of the heat exchanging wall, $\theta_{w}(0)$ and $\theta_{w}\left(\xi_{L}\right)$, is imposed by the fluid entering the heat exchanger in that section, so that we may anticipate that $\theta_{w}(0)=0$ and $\theta_{w}\left(\xi_{L}\right)=1$.

The solution to the problem stated by Eqs. (1)-(6) provides the temperature field, $\theta_{i}\left(\xi, y_{i}\right)$, along with the plate temperature, $\theta_{w}(\xi)$, for given values of $m, k$, and $\xi_{L}$. Moreover, it provides the local heattransfer rate, $v(\xi)$, defined in Eq. (4), which represents the dimensionless heat flux from fluid 2 to fluid 1 , as well as the outlet temperature profiles $\theta_{1}=\theta_{1, \text { out }}\left(y_{1}\right)$ at $\xi=\xi_{L}, \quad 0 \leqslant y_{1}<1$,

$\theta_{2}=\theta_{2, \text { out }}\left(y_{2}\right)$ at $\xi=0, \quad 0 \leqslant y_{2}<1$.

Another interesting result is the distribution of the bulk, or mixingcup, temperatures of both streams

$\theta_{m i}(\xi)=\int_{0}^{1} \frac{3}{2}\left(1-y_{i}^{2}\right) \theta_{i}\left(\xi, y_{i}\right) d y_{i}$

defined as the uniform temperature that would eventually be attained if the fluid at a particular section $\xi$ was allowed to evolve adiabatically.

Following Nunge and Gill $[41,42]$, the analytical solution of the problem can be written as an infinite series of eigenfunctions

$\theta_{i}\left(\xi, y_{i}\right)=A+\sum_{n=-\infty}^{+\infty} C_{n} e^{-\lambda_{n} \xi} f_{n, i}\left(y_{i}\right)$

where satisfaction of the inlet boundary conditions at both ends of the heat exchanger forces us to use an infinite set of both positive $(n>0)$ and negative $(n<0)$ eigenvalues, $\lambda_{n}$, and their corresponding eigenfunctions, $f_{n, i}\left(y_{i}\right)$. The orthogonality condition for the eigenfunctions can then be used, together with the inlet conditions (5) and (6), to calculate the expansion coefficients $A$ and $C_{n}$ [52]. However, the convergence of the series becomes very slow at small axial distances from the inlets, $\xi \ll 1$ (or, alternatively, $\xi_{L}-\xi \ll 1$ ), where a large number of terms is required for an accurate description of the solution $[41,45]$. Accordingly, as the inlets are approached the calculation becomes tedious and the numerical solution may not even converge. It is therefore necessary to consider an alternative method of solution for the counterflow heat exchanger in this region. The alternative approach, due to Lévêque [8], exploits the fact that for sufficiently large Peclet numbers the temperature variations are initially confined to a thin layer of fluid adjacent to the wall where the velocity profile is linear - corresponding to the near-wall velocity gradient of the fully developed flow (see also Refs. $[4,9,13,32])$.

In this paper we shall extend the classical Lévêque analysis to the conjugate fluid-fluid problem that arises in the thermal entrance region of counterflow heat exchangers. Thus, in the limiting case, $\operatorname{Pe}_{i} \gg 1, \operatorname{Pr}_{i} \gg 1$, that we are analyzing, the flow in the heat exchanger is under thermal development conditions. Hence, at distances from the inlets that are small compared to the thermal entry length, of characteristic size $\xi \sim \lambda_{1}^{-1}$ (or, alternatively, $\left.\xi_{L}-\xi \sim\left|\lambda_{-1}^{-1}\right|\right)$, the temperature field presents a thermal boundary layer structure in the vicinity of the separating plate. The special 
feature of the counter-current configuration considered here is that due to the different sign of the flow velocity at both sides of the separating plate the thermal boundary layer adopts the form of a self-similar solution of the second kind [58], to be discussed in the following sections.

\section{The self-similar thermal entrance region for small axial distances}

In the following sections we shall focus our attention on the analysis of the thermal entrance region of fluid 1 . As discussed in the Appendix, the resulting expressions can be used, together with appropriate symmetry conditions, to describe the thermal entrance region of fluid 2 .

When written in terms of the new independent variables $\eta_{i}=\left(1-y_{i}\right) / \xi^{1 / 3}$, Eqs. (1) and (2) take the approximate form

$\frac{3}{2} \eta_{1} \xi^{1 / 3}\left(\frac{\partial \theta_{1}}{\partial \xi}-\frac{\eta_{1}}{3 \xi} \frac{\partial \theta_{1}}{\partial \eta_{1}}\right)=\frac{1}{\xi^{2 / 3}} \frac{\partial^{2} \theta_{1}}{\partial \eta_{1}^{2}}$

$-m \frac{3}{2} \eta_{2} \xi^{1 / 3}\left(\frac{\partial \theta_{2}}{\partial \xi}-\frac{\eta_{2}}{3 \xi} \frac{\partial \theta_{2}}{\partial \eta_{2}}\right)=\frac{1}{\xi^{2 / 3}} \frac{\partial^{2} \theta_{2}}{\partial \eta_{2}^{2}}$,

in $0<\xi \ll 1,0<\eta_{i}<\infty$, where we have approximated the fully developed velocity profile $(3 / 4)\left(2-\eta_{i} \xi^{1 / 3}\right) \eta_{i} \xi^{1 / 3}$ by the linear velocity profile $(3 / 2) \eta_{i} \xi^{1 / 3}$ that prevails in the near-wall region, thus neglecting terms of order $\xi^{1 / 3}$ in the convective terms. Hereafter the problem stated by Eqs. (11) and (12) will be referred to as the inner problem, in contraposition to the original problem, which will be referred to as the outer problem.

Assuming separation of variables of the form

$\theta_{i}\left(\xi, \eta_{i}\right)=\theta_{w}(\xi) \Theta_{i}\left(\eta_{i}\right)$

we obtain the temperature variation along the heat exchanging plate as

$\theta_{w}(\xi)=\Theta_{w 1} \xi^{j}$

where the exponent $j$ has yet to be determined and $\Theta_{w 1}$ is a constant of order unity to be calculated by matching the inner (i.e. Lévêque) self-similar solution with the numerical solution of the outer problem for $\xi \ll 1$. Substituting expressions (13) and (14) in (11) and (12) and absorbing the parameter $m$ into the new stretched variable $\hat{\eta}_{2}=m^{1 / 3} \eta_{2}$, we obtain the following differential equations for the self-similar temperature profiles $\Theta_{1}\left(\eta_{1}\right)$ and $\Theta_{2}\left(\hat{\eta}_{2}\right)$

$\frac{3}{2} \eta_{1}\left(-j \Theta_{1}+\frac{\eta_{1}}{3} \frac{\partial \Theta_{1}}{\partial \eta_{1}}\right)+\frac{\partial^{2} \Theta_{1}}{\partial \eta_{1}^{2}}=0$,

$-\frac{3}{2} \hat{\eta}_{2}\left(-j \Theta_{2}+\frac{\hat{\eta}_{2}}{3} \frac{\partial \Theta_{2}}{\partial \hat{\eta}_{2}}\right)+\frac{\partial^{2} \Theta_{2}}{\partial \hat{\eta}_{2}^{2}}=0$

to be solved in $0<\eta_{1}<\infty, 0<\eta_{2}<\infty$ subject to the boundary conditions stating the continuity of temperatures and heat fluxes at the heat exchanging wall

$\Theta_{1}=\Theta_{2}=1, \quad \frac{\partial \Theta_{1}}{\partial \eta_{1}}=-\hat{k} \frac{\partial \Theta_{2}}{\partial \hat{\eta}_{2}} \quad$ at $\quad \eta_{1}=\hat{\eta}_{2}=0$

and the matching conditions with the solution of the outer problem at the edges of the thermal boundary layers

$\Theta_{1} \rightarrow 0$ as $\eta_{1} \rightarrow \infty$

$-j \Theta_{2}+\frac{\hat{\eta}_{2}}{3} \frac{\partial \Theta_{2}}{\partial \hat{\eta}_{2}} \rightarrow 0 \quad$ as $\quad \hat{\eta}_{2} \rightarrow \infty$

or, alternatively, $\Theta_{2} \sim C \hat{\eta}_{2}^{3 j}$ as $\hat{\eta}_{2} \rightarrow \infty$, where $C$ is a constant to be determined as part of the solution.

Although the matching condition for $\Theta_{1}$ is obvious, the one for $\Theta_{2}$ deserves further attention. Specifically, condition (19) must be satisfied since otherwise the second derivative $\partial^{2} \Theta_{2} / \partial \hat{\eta}_{2}^{2}$ in Eq. (16) would tend to infinity as $\hat{\eta}_{2} \rightarrow \infty$, thus preventing the selfsimilar solution to match with the solution of the outer problem.

It is interesting to note that the lumped parameter

$\hat{k}=m^{1 / 3} k=\left(\frac{U_{2}}{U_{1}} \frac{a_{1}}{a_{2}} \frac{\alpha_{1}}{\alpha_{2}}\right)^{1 / 3} \frac{k_{2}}{k_{1}}=\left(\frac{\mathcal{A}_{2}}{\mathcal{A}_{1}} \frac{\alpha_{1}}{\alpha_{2}}\right)^{1 / 3} \frac{k_{2}}{k_{1}}$,

appearing in the condition of continuity of heat fluxes (17) is the only dimensionless parameter that remains in the problem. As shown by the last equality in Eq. (20), this parameter can be rewritten in terms of the ratio, $\mathcal{A}_{2} / \mathcal{A}_{1}$, of the wall velocity gradients, $\mathcal{A}_{i}=\left.\left(\partial u_{i} / \partial \mathrm{y}_{i}\right)\right|_{\mathrm{y}_{i}=1}$, of fluids 1 and 2 , which becomes the only relevant fluid-dynamical parameter of the problem. We would also like to mention that $\hat{k}=m^{1 / 3} k$ is the only parameter that emerges in the asymptotic description of the higher order eigenvalues $\left(\lambda_{ \pm n}\right.$ for $n \rightarrow \infty)$ of the original problem (1)-(6), whose corresponding eigenfunctions are those mainly involved in the mathematical description of the thermal entrance region [52].

\section{The self-similar solution}

For a given value of $\hat{k}$ the self-similar problem stated above admits a solution for a single value of $j$, which must be determined as an eigenvalue. Accordingly, the temperature field in the thermal entrance region has the character of a self-similar solution of the second kind [58], where the exponent $j$ cannot be obtained from conservation laws, but only by solving the nonlinear eigenvalue problem given by Eqs. (15)-(19).

The solution to this problem can be obtained in closed analytical form in terms of Whittaker's confluent hypergeometric functions $[59,60]$ using standard symbolic algebra packages, such as Mathematica or Maple, leading to

$\Theta_{1}\left(\eta_{1}, j\right)=A \eta_{1}^{-1} \exp \left(-\eta_{1}^{3} / 12\right) \Gamma(1+j) W_{\kappa_{1}(j), \mu}\left(\eta_{1}^{3} / 6\right)$,

$\Theta_{2}\left(\hat{\eta}_{2}, j\right)=A \hat{\eta}_{2}^{-1} \exp \left(\hat{\eta}_{2}^{3} / 12\right) \Gamma(1 / 3-j) W_{k_{2}(j), \mu}\left(\hat{\eta}_{2}^{3} / 6\right)$,

with $A=3^{1 / 2} 6^{1 / 3} \Gamma(2 / 3) /(2 \pi), \kappa_{2}(j)=-\kappa_{1}(j)=1 / 3+j$, and $\mu=1 / 6$. In the above expressions the value of $j$ is given implicitly as a function of $\hat{k}$ by the condition of continuity of heat fluxes (17), which, applying symbolic derivation to (21) and (22) and particularizing the results at $\eta_{1}=\hat{\eta}_{2}=1$, reduces to the closed-form transcendental equation

$\hat{k}=\frac{j \Gamma(j) \Gamma(-j)}{(1 / 3-j) \Gamma(1 / 3-j) \Gamma(-1 / 3+j)}$,

written here in terms of the Gamma function $\Gamma(z)$. The variation of $j$ with $\hat{k}$ given by this expression is plotted in Fig. 2(a), which shows a monotonic decrease in the exponent $j$ from $1 / 3$ to 0 as $\hat{k}$ grows from 0 to $\infty$. Notice, in particular, that the logarithmic scale used for $\hat{k}$ renders the curve $j(\hat{k})$ antisymmetric with respect to $\hat{k}=1, j=$ $1 / 6$, due to the invariance of Eq. (23) under the transformation $\hat{k} \leftrightarrow \hat{k}^{-1}, j \leftrightarrow 1 / 3-j$.

Fig. 3 shows the self-similar temperature profiles given by Eqs. (21)-(23) corresponding to different values of $\hat{k}$. The asymptotic behavior $\Theta_{2} \simeq C \hat{\eta}_{2}^{3 j}$ as $\hat{\eta}_{2} \rightarrow \infty$ is also plotted in the figure, where the value of the constant $C=\left[3^{1 / 2} \Gamma(2 / 3) \Gamma(1 / 3-j)\right] /\left(6^{j} 2 \pi\right)$ results from the application of the asymptotic expansion $W_{\kappa, \mu}(z) \propto z^{\kappa} \exp$ $(-z / 2)\left[1+\mathcal{O}\left(z^{-1}\right)\right]$ as $|z| \rightarrow \infty$ to Eq. (22). This asymptotic behavior holds in the matching region $(\xi / m)^{1 / 3} \ll\left(1-y_{2}\right) \ll 1$ between the inner (Lévêque) and outer (numerical) solutions, and can be rewritten in terms of outer variables as follows

$\theta_{2}\left(\xi, y_{2}\right)=\Theta_{w 1} \xi \mathrm{C} \hat{\eta}_{2}^{3 j}=\Theta_{w 1} C m^{j}\left(1-y_{2}\right)^{3 j}$.

Once we have obtained the solution, we can evaluate the local heat-transfer rate 

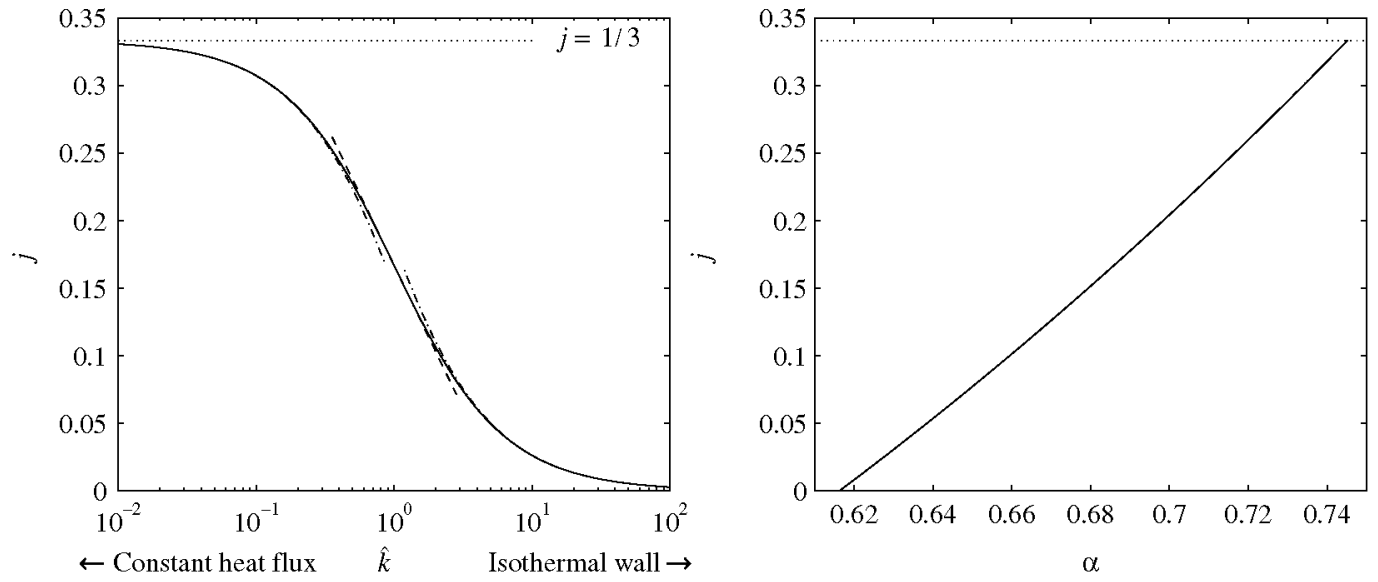

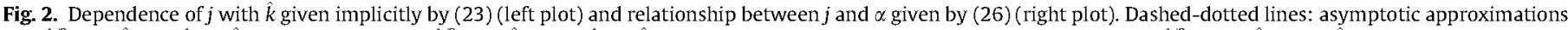
$j \simeq 3^{1 / 2} \pi^{-1}(2 \hat{k}+1)^{-1}$ for $\hat{k} \gg 1$, and $j \simeq 1 / 3-3^{1 / 2} \pi^{-1}\left(2 \hat{k}^{-1}+1\right)^{-1}$ for $\hat{k} \ll 1$; dashed lines: asymptotic approximation $j \simeq\left[1-3^{1 / 2} \pi^{-1} \log \hat{k}\right] / 6$ for $|\hat{k}-1| \ll 1$.

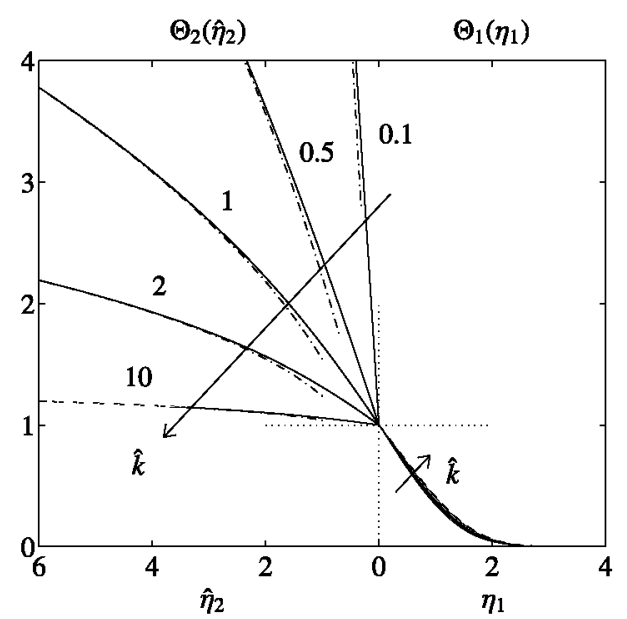

Fig. 3. Self-similar temperature profiles $\Theta_{1}\left(\eta_{1}\right)$ and $\Theta_{2}\left(\hat{\eta}_{2}\right)$ given by $(21)-(23)$ for different values of $\hat{k}=m^{1 / 3} k$. Dashed-dotted lines: asymptotic approximation $\Theta_{2} \sim C \hat{\eta}_{2}^{3 j}$ as $\hat{\eta}_{2} \rightarrow \infty$, with $C=\left[3^{1 / 2} \Gamma(2 / 3) \Gamma(1 / 3-j)\right] /\left(6^{j} 2 \pi\right)$; dashed lines: asymptotic profile $\Theta_{1}\left(\eta_{1}\right)$ corresponding to $\hat{k} \rightarrow \infty$ (isothermal wall limit).

$v(\xi)=\left.\frac{\partial \theta_{1}}{\partial y_{1}}\right|_{y_{1}=1}=\left.\Theta_{w 1} \xi^{j} \frac{\partial \eta_{1}}{\partial y_{1}} \frac{\partial \Theta_{1}}{\partial \eta_{1}}\right|_{\eta_{1}=0}=\Theta_{w 1} \alpha(j) \xi^{j-1 / 3}$

where

$\alpha(j)=\left.\frac{\partial \Theta_{1}}{\partial \eta_{1}}\right|_{\eta_{1}=0}=\frac{3^{1 / 2} 6^{2 / 3}[\Gamma(2 / 3)]^{2}}{4 \pi} \frac{\Gamma(1+j)}{\Gamma(2 / 3+j)}$,

is a bounded function that takes values of order unity, $0.6163 \leqslant \alpha \leqslant 0.7452$ for $0 \leqslant j \leqslant 1 / 3$, as shown in Fig. 2 (b). It is interesting to note that Eq. (25) predicts a singular behavior for the heattransfer rate, $v(\xi) \sim \xi^{j-1 / 3}$, as long as $\hat{k}>0(j<1 / 3)$. Note also the weak dependence of $\Theta_{1}\left(\eta_{1}\right)$ on $\hat{k}$, which results in an almost constant value of $\alpha$.

We would like to point out that the uniparametric family of solutions presented above constitutes a natural extension to the classical first and second Lévêque solutions, which are recovered for large and small values of $\hat{k}$ respectively. Thus, in the limit $\hat{k} \rightarrow \infty \quad(j \rightarrow 0)$ we obtain the isothermal wall solution: $\theta_{w}(\xi) \equiv 1, \Theta_{1}\left(\eta_{1}, 0\right)=1-\Gamma\left(1 / 3, \eta_{1}^{3} / 6\right) / \Gamma(1 / 3), v(\xi)=0.6163 \xi^{-1 / 3}$, where $\Gamma(a, z)$ denotes the incomplete Gamma function and $\Theta_{w 1}$ is set equal to unity without loss of generality. On the other hand, for $\hat{k}=0(j=1 / 3)$ we recover the constant heat flux solution: $v(\xi) \equiv 1$, $\theta_{w}(\xi)=0.7452^{-1} \xi^{j}$, and $\Theta_{1}\left(\eta_{1}, 1 / 3\right)$ given by Eq. (21), where $\Theta_{w 1}$ is set equal to $0.7452^{-1}$ in order to normalize the dimensionless heattransfer rate to unity.

Integration of Eq. (1) across the channel width using (4) and (25) to evaluate $v(\xi)$ provides the evolution of the mixing-cup temperature of fluid 1

$\theta_{m 1}(\xi)=\int_{0}^{\xi} 2 v(\xi) d \xi=2 \Theta_{w 1} \alpha(j) \frac{\xi^{j+2 / 3}}{j+2 / 3}$

where use has been made of the inlet boundary condition $\theta_{m 1}(0)=0$. According to this expression, the singular heat transfer rates that occur for $0 \leqslant j<1 / 3$ in the thermal entrance region result in an enhanced mixing-cup temperature growth rate for the fluid entering the heat exchanger as compared to the linear growth rate, $\theta_{m 1}(\xi) \sim \xi$, predicted by a constant overall heat transfer coefficient which in the thermal entrance region corresponds, in first approximation, to a constant value of $v(j=1 / 3)$. Accounting for the heat transfer enhancement in the thermal entrance region of laminar counterflow heat exchangers may lead to further improvements in the design and operation of practical heat-transfer devices, particularly of short heat exchangers, where this effect is known to have a larger impact [41].

The self-similar description of the thermal entrance region presented above fails in a small region near the inlet section, close to the heat exchanging plate, where upstream conduction becomes important and must be retained in Eqs. (1) and (2). In this region - whose characteristic length, of order $\mathrm{Pe}_{1}^{-1 / 2} a_{1}$, is small compared to $a_{1}$ when $\mathrm{Pe}_{1} \gg 1$ - upstream conduction smoothes out the singularities that arise in $\theta_{w}(\xi)$ and $v(\xi)$ as $\xi \rightarrow 0$ according to (14) and (25).

The characteristic size of this region can be used to estimate the order of magnitude of the maximum longitudinal temperature gradient suffered by the heat exchanging plate, which is of great practical importance for predicting problems of fouling and thermal stresses in the walls. Thus, a rough calculation from Eq. (14) taking $\xi \sim\left(\mathrm{Pe}_{1}^{-1 / 2} a_{1}\right) /\left(\mathrm{Pe}_{1} a_{1}\right) \sim \mathrm{Pe}_{1}^{-3 / 2}$ provides $d \theta_{w} /\left.d \xi\right|_{\max } \sim \Theta_{w 1} j \mathrm{Pe}_{1}^{3(1-j) / 2}$, or alternatively, in dimensional form,

$\left.\frac{d T_{w}}{d X}\right|_{\max } \sim \frac{T_{2, \text { in }}-T_{1, \text { in }}}{a_{1}} \Theta_{w 1} j \mathrm{Pe}_{1}^{(1-3 j) / 2}$

Fig. 4 shows the dependence of the maximum dimensionless axial temperature gradient, $j \mathrm{Pe}_{1}^{(1-3 i) / 2}$, with both $\hat{k}$ and $\mathrm{Pe}_{1}$. Note that in the plot we ignore the effect of the constant $\Theta_{w 1}$, which accounts for the non-local influence of the parameters $m, k$, and $\xi_{L}$ of the outer problem (see Tables 1 and 2 for details). As expected, in the isothermal wall limit, $\hat{k} \rightarrow \infty$, the longitudinal temperature gradient 


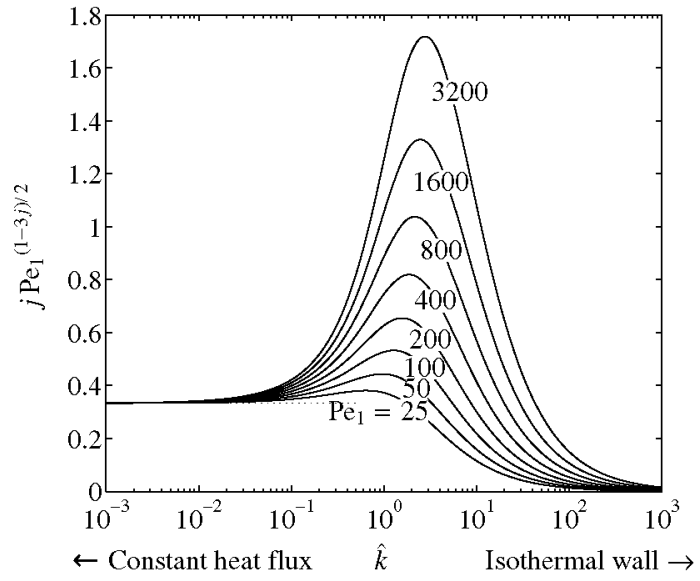

Fig. 4. Characteristic value of the maximum longitudinal wall temperature gradient at the thermal entrance region of fluid $1,\left(d T_{w} / d X\right)_{\max } /\left[\Theta_{w 1}\left(T_{2, \text { in }}-T_{1, \text { in }}\right) /\right.$ $\left.a_{1}\right] \sim j \mathrm{Pe}_{1}{ }^{(1-3 j) / 2}$, as a function of $\hat{k}$ for several values of the Peclet number, $\mathrm{Pe}_{1} \gg 1$. The curves reach the peak value $\mathrm{Pe}_{1}^{\left[(1 / 2)-\left(1 / \log P e_{1}\right)\right]} / \log \mathrm{Pe}_{1}^{3 / 2}$ for $j=1 / \log \mathrm{Pe}_{1}^{3 / 2}$.

decreases asymptotically to zero, while in the constant heat flux limit, $\hat{k} \rightarrow 0$, it approaches the value $1 / 3$ for all $\mathrm{Pe}_{1}$. For intermediate values of $\hat{k}$ the longitudinal temperature gradient grows with $\mathrm{Pe}_{1}$, reaching a peak value $\mathrm{Pe}_{1}^{\left[(1 / 2)-\left(1 / \operatorname{logP} \mathrm{e}_{1}\right]\right]} / \log \mathrm{Pe}_{1}^{3 / 2}$ for $j=1 / \log \mathrm{Pe}_{1}^{3 / 2}$, corresponding to a value of $\hat{k}$ slightly larger than one.

Using order of magnitude estimates in (25) we can also obtain the order of magnitude of the maximum transverse wall temperature gradient, which can be written as

$\left.\left.\frac{d T_{w}}{d Y}\right|_{\max } \sim \frac{k_{1}}{k_{w}} \frac{\alpha(j)}{j} \frac{d T_{w}}{d X}\right|_{\max }$,

in terms of the thermal conductivity of the heat exchanging wall, $k_{w}$.

According to the above discussion, transverse gradients will dominate for poorly conductive plates, $k_{1} / k_{w} \gg 1$, near the isothermal wall limit, $\hat{k} \rightarrow \infty, j \rightarrow 0$. In contrast, longitudinal temperature gradients will be more vigorous for highly conductive plates, $k_{1} /$ $k_{w} \ll 1$, near the constant heat flux limit, $\hat{k} \rightarrow 0, j \rightarrow 1 / 3$.

\section{Numerical solution and discussion of results}

In order to assess the domain of validity of the generalized Lévêque solution, finite-difference numerical solutions of the problem described by Eqs. (1)-(6) were carried out for selected values of the governing parameters $m, k$, and $\xi_{L}$. The governing equations (1) and (2) were discretized using second-order centered finite differences on a distorted grid with nodes clustered near the inlet, outlet and separating wall. Second-order non-centered finite differences were used to discretize the derivatives appearing in the boundary conditions represented by Eqs. (3)-(6). The steady solution was obtained using a time-marching method with local time stepping until the convergence criteria $|\mathcal{R}|<10^{-5}$ was achieved, $|\mathcal{R}|$ being a suitable norm of the residuals (e.g., the infinite norm). Further details on the numerical method can be found elsewhere $[52,61]$.

The numerical solutions were used to calculate the value of $\Theta_{w 1}$ by matching the profiles $\theta_{w}(\xi), v(\xi), \theta_{m 1}(\xi)$, and $\theta_{2, \text { out }}\left(y_{2}\right)$, to the self-similar expressions given by Eqs. (14), (23)-(27) at the thermal
Table 2

Numerical values of $\Theta_{w 1}$ and $\Theta_{w 2}$ for five benchmark cases corresponding to $\xi_{L}=0.0625$ and selected values of $m$ and $k$. The estimated numerical error of $\Theta_{w 1}$ and $\Theta_{w 2}$ is below $1 \%$.

\begin{tabular}{llllll}
\hline$m$ & 1 & 1 & 1 & 2 & 4 \\
\hline$k$ & 1 & 2 & 4 & 1 & 1 \\
\hline$\Theta_{w 1}$ & 0.800 & 0.931 & 0.976 & 0.861 & 0.905 \\
$\Theta_{w 2}$ & 0.800 & 0.590 & 0.376 & 0.831 & 0.891 \\
\hline
\end{tabular}

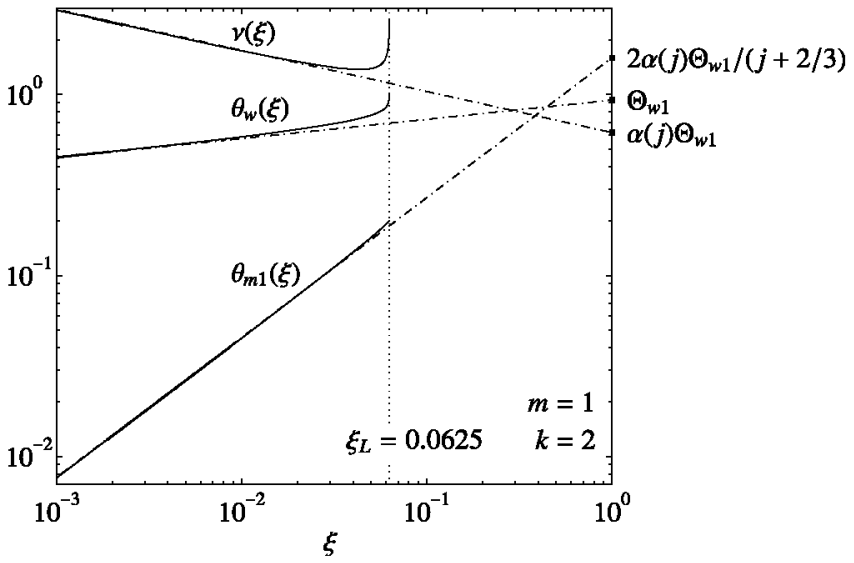

Fig. 5. $\log -\log$ representation of $\theta_{w}(\xi), v(\xi)$, and $\theta_{m 1}(\xi)$ corresponding to $m=1$, $k=2$, and $\xi_{L}=0.0625$. Solid lines: numerical results; dash-dotted lines: asymptotic behavior predicted by the generalized Lévêque solution, Eqs. (14), (25), and (27), with $\Theta_{w 1}=0.931$. Thick solid lines indicate the respective fitting intervals.

entrance region of fluid 1 . To illustrate the matching procedure we may refer to the results presented in Fig. 5, corresponding to $m=1$, $k=1$, and $\xi_{L}=0.0625$. The figure shows the variation of the plate temperature, $\theta_{w}(\xi)$, the local heat transfer rate, $v(\xi)$, and the mixing-cup temperature of fluid $1, \theta_{m 1}(\xi)$, as a function of the distance $\xi$ from the inlet of fluid 1. By taking logarithms on both sides of Eq. (14) we get

$\log \theta_{w}=j \log \xi+\log \Theta_{w 1}$.

Therefore, when plotted on a log-log scale, $\theta_{\mathcal{w}}(\xi)$ should approach a straight line with slope $j$ for $\xi \ll 1$, as confirmed by Fig. 5. To find the corresponding value of $\Theta_{w 1}$ we fitted the plot of $\theta_{w}(\xi)$ to a straight line on different fitting intervals, $\xi_{\min } \leqslant \xi \leqslant \xi_{\max } \ll 1$, and selected the interval which provided the best approximation for the slope $j$. The resulting linear fit was continued until it intercepted the axis $\log \xi=0$, where we read the value of $\log \Theta_{w 1}$. The same procedure was applied to match the numerical results to the selfsimilar expressions (24), (25), and (27), leading in all cases to very close values of $\Theta_{w 1}$, the small numerical discrepancies being due to the inability of the numerical method to capture the singular behavior of the solution for small axial distances. The different values of $\Theta_{w 1}$ thus obtained were conveniently averaged to minimize the numerical error, leading to the final results presented in Tables 1 and 2, which correspond to the different benchmark cases considered below.

Several conclusions may be drawn from the results collectively shown in Fig. 5 and Tables 1 and 2. First of all, the generalized Lévêque solution is seen to accurately describe the solution up to distances of the order of the thermal development length away

Table 1

Numerical values of $\Theta_{w 1} \equiv \Theta_{w 2}$ for five benchmark cases corresponding to $m=k=1$ and selected values of $\xi_{I}$. The estimated numerical error of $\Theta_{w 1}$ and $\Theta_{w 2}$ is below $1 \%$.

\begin{tabular}{|c|c|c|c|c|c|c|c|c|c|}
\hline$\xi_{L}$ & 0.0625 & 0.125 & 0.25 & 0.375 & 0.5 & 0.625 & 0.75 & 0.875 & 1 \\
\hline$\Theta_{w 1} \equiv \Theta_{w 2}$ & 0.800 & 0.698 & 0.580 & 0.498 & 0.437 & 0.390 & 0.351 & 0.320 & 0.293 \\
\hline
\end{tabular}



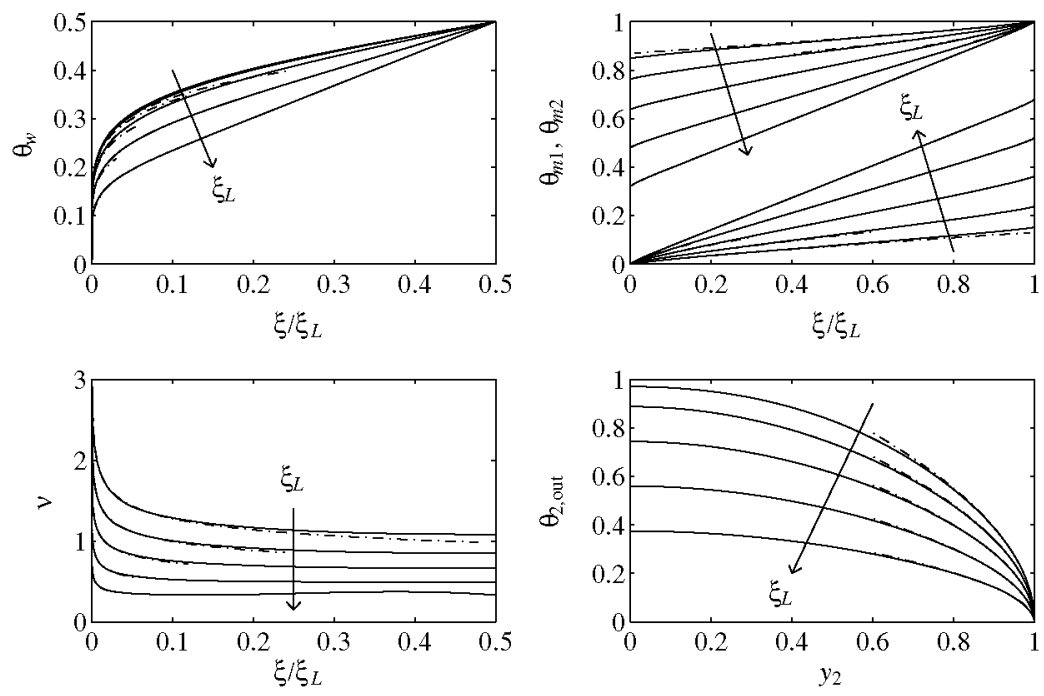

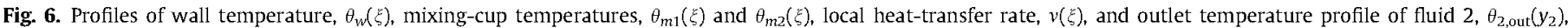

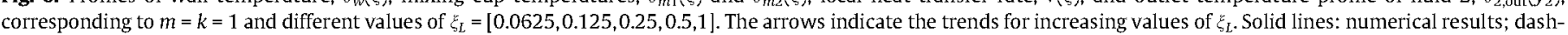

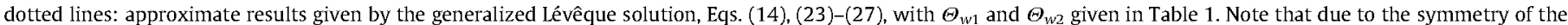
problem for $m=k=1 \theta_{w}(\xi)$ and $v(\xi)$ are only plotted for $0 \leqslant \xi \leqslant \xi_{L} / 2$.

from the inlets, $\xi \sim \lambda_{1}^{-1} \sim 0.02$, where $\lambda_{1} \sim 25-40$ denotes the first positive eigenvalue of the problem modeled by Eqs. (1)-(6) (see [52] for details). This result is indeed rather general as demonstrated by a detailed inspection of the solutions corresponding to the benchmark cases listed in Tables 1 and 2 . Second, the value of $\Theta_{w 1}$ is seen to decrease with the heat exchanger length, $\xi_{L}$, and increase both with $m$ and - more appreciably - with $k$. And third, the estimated numerical error associated with the values of $\Theta_{w 1}$ and $\Theta_{w 2}$ is quite large, about $1 \%$. This is a direct consequence of the singular character of the solution in the thermal entrance region, which is hard to capture using standard finite-difference methods. Thus, high-order methods would be mandatory to calculate $\Theta_{w 1}$ and $\Theta_{w 2}$ with better accuracy. However, the aim of this work was not to provide precise numerical values of these constants (which are, in addition, configuration dependent), but to unveil the general structure of the solution in the thermal entrance region of laminar counterflow heat exchangers.
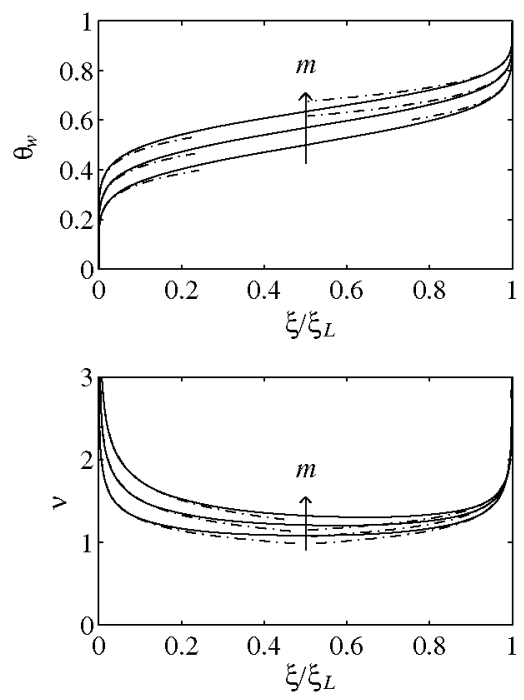

For illustrative purposes, Fig. 6 shows the variations of the wall temperature, $\theta_{w}$, mixing-cup temperatures, $\theta_{m 1}$ and $\theta_{m 2}$, and local heat-transfer rate, $v$, as a function of $\xi$, along with the outlet temperature profile of fluid $2, \theta_{2, \text { out }}\left(y_{2}\right)$, corresponding to $m=k=1$ and different values of $\xi_{L}=[0.0625,0.125,0.25,0.5,1]$. The approximate profiles predicted by the self-similar thermal entrance solution through expressions (14), (24), (25), and (27) are also shown for illustrative purposes. As can be seen, the self-similar near-inlet solution describes accurately the asymptotic behaviors of the wall temperature, $\theta_{w}$, and local heat-transfer rate, $v$, as the inlet is approached, and provides a fairly good prediction of the mixingcup temperatures of both streams, $\theta_{m 1}$ and $\theta_{m 2}$, over a large portion of the heat exchanger length. It also provides a precise description of the singularity that appears in the outlet temperature profile of the fluid exiting the heat exchanger.

Parallel results are presented in Figs. 7 and 8, where we fix the heat exchanger length, $\xi_{L}=0.0625$, and vary the values of

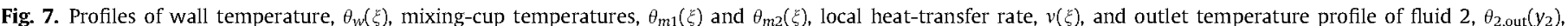

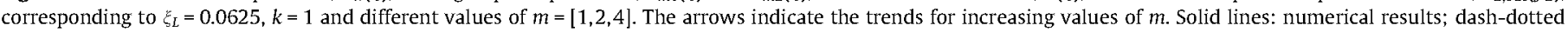
lines: approximate results given by the generalized Lévêque solution, Eqs. (14), (23)-(27) with $\Theta_{w 1}$ and $\Theta_{w 2}$ given in Table 2. 

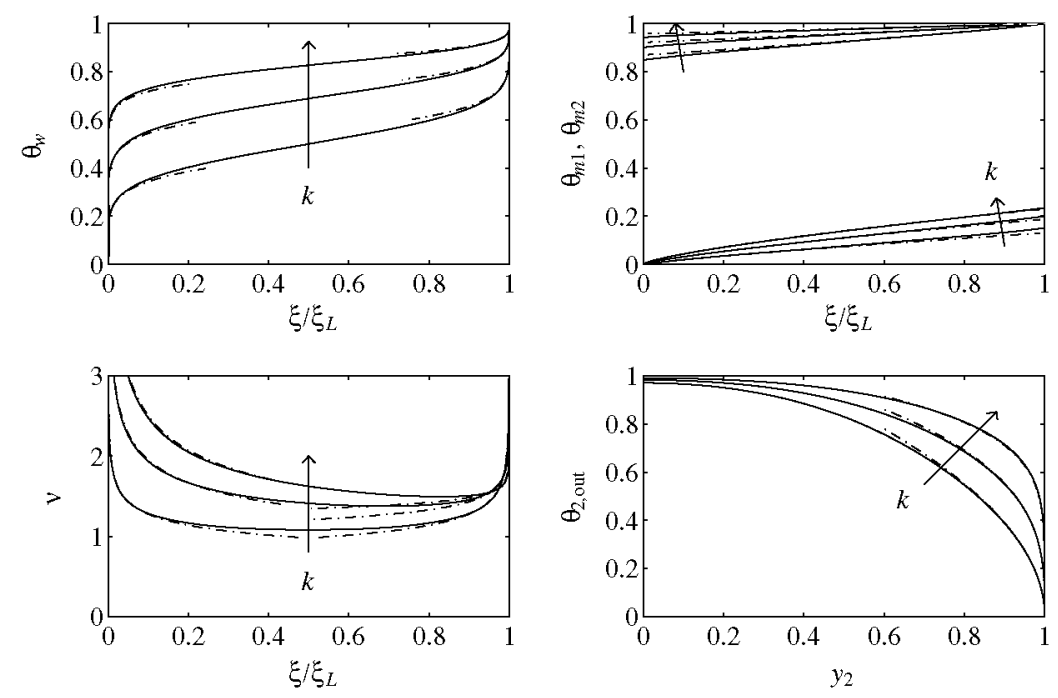

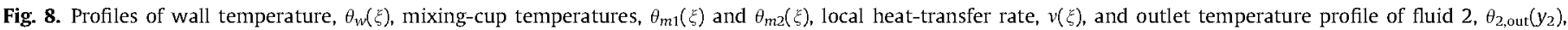

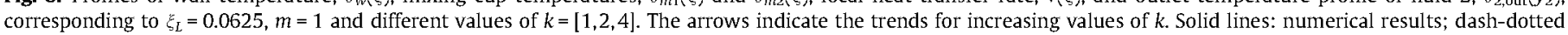
lines: approximate results given by the generalized lévêque solution, Eqs. (14), (23)-(27) with $\Theta_{w 1}$ and $\Theta_{w 2}$ given in Table 2.

$m=[1,2,4]$ and $k=[1,2,4]$. Note that the agreement between the numerical and the theoretical predictions is excellent in all cases.

\section{Conclusions}

In this paper we have combined analytical, symbolic, and numerical techniques to investigate the structure of the conjugate fluid-fluid problem that arises in the thermal entrance region of fully-developed laminar counterflow heat exchangers. The study, carried out for constant property fluids, assumes that the Prandtl and Peclet numbers are both large compared to unity, and neglects axial conduction both in the fluids and in the plate, assumed to be thermally thin. The discussion begins with the formulation of the conservation equations and boundary conditions governing the temperature field in parallel-plate counterflow heat exchangers, but attention is rapidly shifted to the self-similar description of the thermal entrance region for small axial distances.

The solution of the resulting mathematical problem has the character of a self-similar solution of the second kind and can be expressed in terms of Whittaker's confluent hypergeometric functions. According to this local, near-inlet solution, the dimensionless plate temperature grows as $\xi^{j}$, where $\xi$ is the dimensionless distance measured from the inlet section, and the exponent, $0 \leqslant j \leqslant 1 / 3$, must be calculated as part of the solution as an eigenvalue. Indeed, the analysis demonstrates that the exponent $j$ depends exclusively on the lumped parameter $\hat{k}$, defined by Eq. (20) in terms of the ratios of the wall velocity gradients, thermal diffusivities, and thermal conductivities of the fluids entering and exiting the heat exchanger. Interestingly, the generalized Lévêque solution presented here admits as limiting cases the classical isothermal wall (i.e. first) and constant heat-flux (i.e. second) Lévêque solutions, obtained respectively for large and small values of $\hat{k}$.

As a result of the study, closed-form analytical expressions for the self-similar near-inlet temperature distributions, local heattransfer rates, and bulk temperature distribution of the fluid entering the heat exchanger were obtained. The analytical expressions were defined up to an undetermined constant which has to be obtained by matching with the (outer) numerical solution. Thereafter, these expressions were compared with fully numerical simulations, showing very good agreement in the counterflow parallelplate configuration in all cases.
The local character of the generalized Lévêque solution makes it applicable to more complex configurations extensively used in industrial applications, such as concentric tube heat exchangers. Using the appropriate value of the ratio of the wall velocity gradients, $\mathcal{A}_{2} / \mathcal{A}_{1}$, to determine the value of $\hat{k}$ in Eq. (20), the analytical expressions presented herein would provide a reliable first-order approximation for the quasi-planar temperature field in the thermal entrance region of concentric tube heat exchangers. Since the effect of curvature is known to be significant, the resulting approximation should be used with caution in cylindrical geometries because it is expected to be less accurate than for the planar case considered here [13].

Further improvements of the generalized Lévêque solution would be worthwhile exploring. For instance, the analysis could be extended to cover finite wall conduction effects, curvature effects (by consideration of cylindrical configurations), nonlinear inlet velocity profiles, swirling flows, or non-Newtonian fluids. Besides its value as a dependable solution to a fundamental problem, the analytical solution presented in this work may find application in benchmarking exercises of numerical codes.

\section{Acknowledgements}

This work was partially supported by Projects S-505/ENE/0229 of the Spanish Comunidad de Madrid and ENE2008-06683-C03-02 of the Spanish Ministerio de Ciencia e Innovación.

\section{Appendix A. The self-similar entrance region of fluid 2}

The self-similar description of the thermal entrance region of fluid 1 presented in Section 3 can be extended using appropriate symmetry conditions to describe the self-similar entrance region of fluid 2. First of all, we should note that the problem formulated by Eqs. (1)-(6) is invariant under the group of transformations $1 \leftrightarrow 2, \theta_{i} \leftrightarrow 1-\theta_{i}, m \leftrightarrow m^{-1}, k \leftrightarrow k^{-1}, \xi \leftrightarrow m^{-1}\left(\xi_{L}-\xi\right)$. This, in turn, ensures the invariance of the self-similar solution of the thermal entrance region under the group of transformations $\hat{k} \leftrightarrow \hat{k}^{-1}, j \leftrightarrow 1 / 3-j, \eta_{i} \leftrightarrow m^{1 / 3} \eta_{i}$. Applying these symmetry conditions in the asymptotic expressions for $\theta_{w}(\xi), v(\xi)$, and $\theta_{m 1}(\xi)$, the respective Eqs. (13), (25), and (27) yield the corresponding expressions for the thermal entrance region of fluid 2 , 
$\theta_{w}(\xi) \simeq 1-\Theta_{w 2}\left(\frac{\xi_{L}-\xi}{m}\right)^{1 / 3-j}$

$v(\xi) \simeq \Theta_{w 2} k \alpha(1 / 3-j)\left(\frac{\xi_{L}-\xi}{m}\right)^{-j}$,

$\theta_{m 2}(\xi) \simeq 1-2 \Theta_{w 2} \frac{\alpha(1 / 3-j)}{1-j}\left(\frac{\xi_{L}-\xi}{m}\right)^{1-j}$

and, in analogy with (24), the asymptotic temperature profile of fluid 1 at the outlet

$\theta_{1}\left(\xi, y_{1}\right) \simeq 1-\Theta_{w 2} C m^{j-1 / 3}\left(1-y_{1}\right)^{1-3 j}$

In (31)-(34) the constant $\Theta_{w 2}$ must be determined by matching with the numerical solution for $\xi_{L}-\xi \ll 1$ following the same procedure applied to $\Theta_{w 1}$.

As can be observed in Figs. 6-8, the resulting approximations for $\theta_{w}(\xi), v(\xi)$, and $\theta_{m 2}(\xi)$ in the thermal entrance region of fluid 2 are also in very good agreement with the numerical results. Although the asymptotic behavior of $\theta_{1}\left(\xi, y_{1}\right)$ is not shown in the figures, the agreement was also found to be satisfactory.

[1] L. Graetz, Ueber die Wärmeleitungsfähigkeit von Flüssigkeiten, Part 1, Ann. Phys. Chem. 18 (1883) 79-94.

[2] L. Graetz, Ueber die Wärmeleitungsfähigkeit von Flüssigkeiten, Part 2, Ann. Phys. Chem. 25 (1885) 337-357.

[3] W. Nusselt, Die Abhangigkeit der Warmeübergangzahl von der Rohrlange, Zeitschr. d. Ver. Deutsch. Ing. 54 (1910) 1154-1158.

[4] R.K. Shah, A.L. London, Laminar flow forced convection in ducts, in: A Source Book for Compact Heat Exchanger Analytical Data, Academic Press, New York, 1978. pp. 196-207.

[5] R.K. Shah, M.S. Bhatti, Laminar convective heat transfer in ducts, in: S. Kakac, R.K. Shah, W. Aung (Eds.), Handbook of Single-Phase Convective Heat Transfer, John Wiley\& Sons, New York, 1987. pp. 3.1-3.137.

[6] W.M.M. Kays, E. Crawford, Convective Heat and Mass Transfer, Third ed., McGrawHill, New York, 1993.

[7] S. Kakaç, Y. Yener, Convective Heat Transfer, CRC Press, Boca Raton, 1995.

[8] M.A. Lévêque, Les lois de la transmission de chaleur par convection, Ann. Mines Memories (12-13) (1928) 201-299. pp. 305-362, 381-415.

[9] R.B. Bird, W.E. Steward, E.N. Lightfoot, Transport Phenomena, John Wiley, New York, 1960 . pp. 363-364.

[10] A.M. Mercer, The growth of the thermal boundary layer in laminar flow between parallel flat plates, Appl. Sci. Res. Sec. A 8 (1959) 357-365.

[11] A.M. Mercer, The growth of the thermal boundary layer at the inlet of a circular tube, Appl. Sci. Res. Sec. A 9 (1960) 450-456.

[12] J. Newman, Extension of Lévêque solution, J. Heat Transfer 91 (1969) 177-178.

[13] M.R. Doshi, The Generalized Lévêque solution, Chem. Eng. Commun. 3 (1979) 165-174.

[14] T.K. Bhattacharyya, D.N. Roy, Laminar heat transfer in a round tube with variable circumferential or arbitrary wall heat flux, Int J. Heat Mass Transfer 13 (1970) 1057-1060.

[15] H.-T. Lin, Y. -P Shih, Unsteady thermal entrance heat transfer of power-law fluids in pipes and plate slits, Int. J. Heat Mass Transfer 24 (1981) 1531-1539.

[16] Y.P. Shih, J.D. Tsou, Extended Lévêque solutions for heat transfer to power law fluids in laminar flow in a pipe, Chem. Eng. J. 15 (1979) 55-62.

[17] S.M. Richardson, Extended lévêque solutions for flows of power law fluids in pipes and channels, Int. J. Heat Mass Transfer 22 (1979) 1417-1423.

[18] P.R. Johnston, Axial conduction and the Graetz problem for a Bingham plastic in laminar tube flow, Int. J. Heat Mass Transfer 34 (1991) 1209-1217.

[19] P.M. Coelho, F.T. Pinho, P.J. Oliveira, Fully developed forced convection of the Phan-Thien-Tanner fluid in ducts with a constant wall temperature, Int. J. Heat Mass Transfer 45 (2002) 1413-1423.

[20] Y.-P. Shih, C.-C. Huang, S.-Y. Tsay, Extended lévêque solution for laminar heat transfer to power-law fluids in pipes with wall slip, Int. J. Heat Mass Transfer 38 (1995). pp. $403-108$

[21] V.L. Okulov, S. Martemianov, T.o. Murakhtina, Generalization of the Lêvêque problem for mass transfer in swirling flows in the entrance region of a cylindrical section, Doklady Phys. 47 (2002) 685-689.

[22] R.E. Lundberg, P.A. McCuen, W.C. Reynolds, Heat transfer in annulat passages. Hydrodynamically developed flow with arbitrarily prescribed wall temperatures or heat fluxes, Int. J. Heat Mass Transfer 6 (1963) 495-529.

[23] P.M. Worsøe-Schmidt, Heat transfer in the thermal entrance region of circular tubes and annular passages with fully developed laminar flow, Int. J. Heat Mass Transfer 10 (1967) 541-551.

[24] R.J. Nunge, E.W. Porta, R. Bentley, A correlation of local Nusselt numbers for laminar flow heat transfer in annuli, Int. J. Heat Mass Transfer 13 (1970) 927931.
25] M.N. Özişik, M.S. Sadeghipour, Analytic solution for the eigenvalues and coefficients of the graetz problem with third kind boundary condition, Int. J. Heat Mass Transfer 25 (1982) 736-739.

[26] T.L. Perelman, On conjugated problems of heat transfer, Int. J. Heat Mass Transfer 3 (1961) 293-303.

[27] E.J. Davis, W.N. Gill, The effects of axial conduction in the wall on heat transfer with laminar flow, Int. J. Heat Mass Transfer 13 (1970) 459-470.

[28] A.V. Luikov, V.A. Aleksashenko, A.A. Aleksashenko, Analytical methods of solving of conjugate problems in convective heat transfer, Int. J. Heat Mass Transfer 11 (1971) 1017-1056.

[29] S. Mori, M. Sakaribara, A. Tanimoto, Steady heat transfer to laminar flow in circular tube with conduction in the tube wall, Jpn. Res. Heat Transfer 3 (1974) $37-46$.

[30] S. Mori, T. Shinke, M. Sakaribara, Steady heat transfer to laminar flow between parallel plates with conduction in wall, Jpn. Res. Heat Transfer 5 (1976) 17-25.

[31] E. Papoutsakis, D. Ramkrishna, Conjugated Graetz problems. I: general formulism and a class of solid-fluid problems, Chem. Eng. Sci. 36 (1981) 1381-1391.

[32] W.C. Lee, Y.H. Ju, Conjugate Lévêque solution for Newtonian fluid in a parallel plate channel, Int. J. Heat Mass Transfer 29 (1986) 941-947.

[33] M. Faghri, E.M. Sparrow, Simultaneous wall and fluid axial conduction in laminar pipe flow heat transfer, Trans. ASME, Ser. C, J. Heat Transfer $102(1980)$ $58-63$.

[34] A. Pozzi, M. Lupo, The coupling of conduction with forced convection in a plane duct, Int. J. Heat Mass Transfer 32 (1989) 1215-1221.

[35] X. Yin, H.H. Bau, The conjugated Graetz problem with axial conduction, Trans ASME 118 (1996) 482-485.

[36] R.P. Stein, The Graetz problem in concurrent-flow, double-pipe, heat exchangers, Chem. Eng. Prog. Symp. Ser 59 (1965) 76-87.

[37] W.N. Gill, E.W. Porta, R.J. Nunge, Heat transfer in thermal entrance region of cocurrent flow heat exchangers with fully developed laminar flow, Int. J. Heat Mass Transfer 11 (1968) 1408-1412.

[38] M.D. Mikhailov, B.K. Shishedjiev, Coupled at boundary mass or heat transfer in entrance concurrent flow, Int. J. Heat Mass Transfer 19 (1976) 553-557.

[39] G. Pagliarini, G.S. Barozzi, Thermal coupling in laminar flow double-pipe heat exchangers, J. Heat Transfer 113 (1991) 526-534.

[40] P. Plaschko, High Peclet number heat exchange between cocurrent streams, Arch. Appl. Mech. 70 (2000) 597-611.

[41] R.]. Nunge, W.N. Gill, Analysis of heat or mass transfer in some countercurrent flows, Int. J. Heat Mass Transfer 8 (1965) 873-886.

[42] R.J. Nunge, W.N. Gill, An analytical study of laminar counterflow double-pipe heat exchangers, A.l.Ch.E. J. 12 (1966) 279-289.

[43] R. Nunge, E.W. Porta, W.N. Gill, Axial conduction in the fluid streams of multistream heat exchangers, Chem. Eng. Progr. Symp. Ser. 63 (1967) 8091.

[44] S. Mori, M. Kataya, A. Tanimoto, Performance of counterflow, parallel plate heat exchangers under laminar flow conditions, Heat Transfer Eng. 2 (1980) $28-38$

[45] E. Papoutsakis, D. Ramkrishna, Conjugated Graetz problems. II: fluid-fluid problems, Chem. Eng. Sci. 36 (1981) 1393-1399.

[46] F. Scofano Neto, R.M. Cotta, Counterflow double-pipe heat exchanger analysis using a mixed lumped-differential formulation, Int. J. Heat Mass Transfer 35 (1992) 1723-1731.

[47] M. Medina, E. Luna, C. Treviño, Numerical solution of the conjugate heat transfer between forced counterflowing streams, Heat Mass Transfer 30 (1995) 297-302

[48] C. Trevino, A. Espinoza, F. Mendez, Steady-state analysis of the conjugate heat transfer between forced counterflowing streams, J. Thermophys. Heat Transfer 10 (1996) $476-483$

[49] C. Trevino, A. Espinoza, F. Mendez, Asymptotic analysis of the transient conjugate heat transfer process between two forced counterflowing streams, SIAM J. Appl. Math 57 (1997) 577-596.

[50] C. Housiadas, F. Ezquerra-Larrode, Y. Drossinos, Technical note numerical evaluation of the Graetz series, Int. J. Heat Mass Transfer 42 (1999) 30133017.

[51] M.D. Mikhailov, R.M. Cotta, Mixed symbolic-numerical computation of convective heat transfer with slip flow in microchannels, Int. Comm. Heat Mass Transfer 32 (2005) 341-348.

[52] M. Vera, A. Liñán, Laminar counterflow parallel-plate heat exchangers: exact and approximate solutions, Int. J. Heat Mass Transfer 53 (2010) 4885-4898.

[53] A. Campo, On the asymptotic solution of the Graetz-Nusselt problem for short $x \rightarrow 0$ and large $x \rightarrow \infty$ with partial usage of finite differences, Numer. Methods Partial Diff. Equat. 20 (2004).

[54] A. Campo, C.H. Amon, Remarkable improvement of the lêvêque solution for isoflux heating with a combination of the transversal method of lines (TMOL) and a computer-extended Fröbenius power series, Int. J. Heat Mass Transfer 48 (2005) $2110-2116$

[55] J.C. Gottifredi, O.D. Quiroga, A.F. Flores, Heat transfer to Newtonian and nonNewtonian fluids flowing in a laminar regime, Int. J. Heat Mass Transfer 26 (1983) $1215-1220$.

[56] J.C. Gottifredi, A.F. Flores, Extended Lévêque solution for heat transfer to nonNewtonian fluids in pipes and flat ducts, Int. J. Heat Mass Transfer 28 (1985) 903-908.

[57] S.N. Singh, Heat transfer by laminar flow in a cylindrical tube, Appl. Sci. Res. A7 (1958) 325-340. 
[58] G.I. Barenblatt, Scaling, self-similarity, and intermediate asymptotics, in Dimensional Analysis and Intermediate Asymptotics, Cambridge University Press, Cambridge, 1996.

[59] E.T. Whittaker, G.N. Watson, A Course of Modern Analysis, Cambridge University Press, Cambridge, 1952. pp. 337.
[60] M. Abramowitz, I. Stegun (Eds.), Handbook of mathematical functions with formulas, graphs, and mathematical tables, national bureau of standards, Appl. Math. Ser. 55 (1964). chapter 13.

[61] C.A.J. Fletcher, Computational techniques for fluid dynamics, Springer Series in Computational Physics, vol. I and Il, Springer-Verlag, Berlin, 1988. 